\title{
PRODUÇÃO DE PAINÉIS LAMINADOS UNIDIRECIONAIS - LVL COM LÂMINAS DE Schizolobium amazonicum, Eucalyptus saligna E Pinus taeda
}

\author{
Setsuo Iwakiri ${ }^{1}$, Jorge Luis Monteiro de Matos ${ }^{1}$, Juliana Afonso Pinto², Lívia Cássia Viana ${ }^{3}$, Marina Moura de Souza ${ }^{4}$, \\ Rosilani Trianoski², Vanessa Coelho Almeida ${ }^{3}$
}

(recebido: 17 de dezembro de 2009; aceito: 30 de agosto de 2010)

\begin{abstract}
RESUMO: Objetivou-se, nesta pesquisa, avaliar a qualidade dos painéis laminados unidirecionais (LVL) produzidos com lâminas de Schizolobium amazonicum (paricá), Eucalyptus saligna e Pinus taeda. Foram produzidos em laboratório painéis LVL com sete lâminas de 2,0 mm de espessura com diferentes composições utilizando resina fenol-formaldeído. As lâminas de paricá foram préclassificadas em diferentes classes de módulo de elasticidade dinâmico (MOEd) através do uso de "stress wave machine". As lâminas de Eucalyptus saligna e Pinus taeda foram utilizadas nas capas dos painéis com objetivo de reforçar a resistência estrutural dos painéis LVL. Foram avaliadas as propriedades de resistência da linha de cola aos esforços de cisalhamento (RLC) e flexão estática (MOE e MOR, edge e flat). A classificação de lâminas de paricá com base nas classes de MOEd não influenciou de forma significativa nos resultados de resistência da linha de cola, MOE e MOR edge. Para o MOE e MOR flat, a utilização de lâminas com MOEd classe 1, contribuiu de forma significativa para o aumento nos valores médios destas propriedades. Da mesma forma, a utilização de lâminas de Eucalyptus saligna nas faces do painel contribuiu para o aumento nos valores médios de MOE e MOR, edge e flat.
\end{abstract}

Palavras-chave: Lâminas de madeira, paricá, compensados.

\section{PRODUCTION OF LAMINATED VENEER LUMBER LVL USING VENEER OF Schizolobium amazonicum, Eucalyptus saligna AND Pinus taeda}

\begin{abstract}
This research evaluated the quality of laminated veneer lumber - LVL manufactured with veneers of Schizolobium amazonicum (paricá), Eucalyptus saligna and Pinus taeda. The LVL panels were manufactured in the laboratory conditions composed by seven veneers, 2,0 mm thickness, with different structural compositions, using phenol-formaldehyde resin. The veneers of Schizolobium amazonicum- paricá- were pre-classified by using stress wave machine. The veneers of Eucalyptus saligna and Pinus taeda were disposed in the face layer to reinforce the structural strength of LVL panels. The LVL quality was evaluated using glue line shear strength and static bending test (MOE and MOR, edge and flat). Grading of paricá veneers based on MOEd did not affected significantly the results of the glue line shear strength and MOE and MOR edge. For the MOE and MOR flat, the use of veneers of MOEd grade 1 contributed significantly to increasing the average values of these properties. In the same way, using the Eucalyptus saligna veneers on the face of LVL resulted in higher average values of MOE and MOR, edge and flat.
\end{abstract}

Key words: Wood veneers, paricá, plywood.

\section{INTRODUÇÃO}

Preocupados com a imagem negativa da devastação da floresta amazônica e pressões ambientalistas sobre uso industrial de madeiras tropicais sem manejo florestal sustentado, os empresários do setor florestal e madeireiro da Amazônia passaram a investir em implantação de florestas plantadas com espécies nativas da região. Uma dessas espécies é o Schizolobium amazonicum, conhecido como paricá, que demonstrou ser uma excelente alternativa do ponto de vista silvicultural, além de produzir madeira com características tecnológicas favoráveis à produção de lâminas e painéis de madeira (SOCIEDADE BRASILEIRA DE SILVICULTURA - SBS, 2007).

Os plantios em escala comercial de paricá Schizolobium amazonicum Huber ex. Ducke, estão concentrados no estado do Pará, especialmente na região de Paragominas. De acordo com os dados da SBS (2007), estima-se que a área de plantio seja superior a 50 mil ha. A sua madeira apresenta coloração branca e tem densidade

\footnotetext{
${ }^{1}$ Engenheiro Florestal, Professor Dr. em Engenharia Florestal - Departamento de Engenharia e Tecnologia Florestal/DETF - Universidade Federal do Paraná/UFPR - Av. Lothário Meissner, 900 - Jardim Botânico - 80210-170 - Curitiba, PR - setsuo@ufpr.br, jmatos@ufpr.br ${ }^{2}$ Engenheira Industrial Madeireira, Mestrando em Engenharia Florestal - Universidade Federal do Paraná - Av. Lothário Meissner, 900 80210-170 - Curitiba, PR - ju_sebaio@yahoo.com.br, rosillani@gmail.com

${ }^{3}$ Engenheira Florestal, Doutoranda em Engenharia Florestal - Universidade Federal do Paraná - Av. Lothário Meissner, 900 - 80210 170 - Curitiba, PR - licvianna@hotmail.com, vanessaufrrj@yahoo.com.br

${ }^{4}$ Engenheira Florestal, Mestre em Engenharia Florestal - Universidade Federal do Paraná - Av. Lothário Meissner, 900 - 80210 -170 Curitiba, PR - mourafloresta@yahoo.com.br
} 
média de $0,40 \mathrm{~g} / \mathrm{cm}^{3}$. O paricá é uma espécie de madeira tropical que apresenta um rápido crescimento, pode ser implantada em plantios homogêneos ou consorciados, e apresenta vantagens de ser resistente ao ataque de pragas e doenças. A sua produtividade média anual é na faixa de 20 a $30 \mathrm{~m}^{3} / \mathrm{ha} /$ ano, estando na mesma faixa de crescimento das espécies de pinus que é de 25 a $30 \mathrm{~m}^{3} / \mathrm{ha} / \mathrm{ano}$, e superior à teca com 15 a 20 m³/ha/ano.

As limitações relacionadas à baixa densidade da madeira de espécies de florestas plantadas de rápido crescimento, e pequenos diâmetros das árvores, podem ser minimizadas com uso de tecnologias de colagem para fabricação de produtos de madeira reconstituída, como painéis laminados de madeira. Bendsten (1978) afirma que para se alcançar êxito no uso dos recursos florestais, provenientes de reflorestamentos, os conceitos tradicionais devem ser modificados e adaptados às características da matéria-prima disponível.

O painel laminado unidirecional é um produto desenvolvido nos EUA na década de setenta, com denominação comercial de "LVL - laminated veneer lumber". A sua aplicação destina-se principalmente ao uso estrutural como: pisos de carrocerias, vagões de trens, escadas, flanges de vigas em "I", entre outras, em função da maior resistência na direção longitudinal ao plano do painel (IWAKIRI, 2005).

De acordo com Pease (1994), os painéis LVL apresentam as seguintes vantagens em comparação à madeira sólida: (i) maior resistência - a estrutura reconstituída dos painéis com processo de classificação das lâminas pode conferir propriedades de resistência que podem ser calculadas com precisão; (ii) flexibilidade dimensional - os painéis podem ser fabricados com qualquer largura e comprimento desejado; (iii) utilização de grande variedade de espécies florestais.

O princípio de construção do painel laminado unidirecional difere do painel compensado multilaminado, pela disposição das lâminas na mesma direção e não a da laminação cruzada. Dessa forma, a resistência do painel na direção longitudinal será maior, possibilitando seu uso em aplicações que requeiram maior resistência à flexão estática (BALDWIN, 1993; TSOUMIS, 1991). Outro fator importante na produção de painéis estruturais LVL é a aplicação dos conceitos sobre distribuição de tensões nas diferentes camadas. De acordo com Bodig \& Jayne (1982), as lâminas externas contribuem mais sobre o módulo de elasticidade e módulo de ruptura da viga que aquelas lâminas colocadas próximas à linha neutra. Portanto, o posicionamento das lâminas de maior resistência, ou de maior módulo de elasticidade próxima à superfície tornam-se mais eficientes. Matos (1997), afirma no seu estudo sobre painéis LVL de Pinus taeda que, a classificação de lâminas em classes de qualidade, pela determinação não destrutiva do módulo de elasticidade dinâmico, baseado no princípio de aplicação de ondas sonoras, mostrou-se altamente eficaz, contribuindo para o aumento significativo das propriedades de resistência mecânica dos painéis testados. Pio (2002), concluiu no seu estudo que a divisão de lâminas de Eucalyptus grandis selecionadas em três classes de módulo de elasticidade dinâmico, contribuiu para o aumento nas propriedades de resistência mecânica dos painéis LVL.

A relação direta entre a densidade da madeira e suas propriedades mecânicas é amplamente conhecida na literatura. Iwakiri et al. (2002), analisando os efeitos do crescimento radial das árvores de Pinus taeda na densidade das lâminas e resistência mecânica dos painéis compensados, constataram que as lâminas obtidas das partes externas da árvore apresentam maior densidade e os painéis produzidos com essas lâminas dispostas nas capas, apresentaram maiores valores de resistência mecânica.

$\mathrm{Na}$ produção de painel laminado unidirecional utiliza-se a resina fenol-formaldeído, por ser a mais indicada para fins estruturais em função de suas características de maior resistência e durabilidade em condições extremas de exposição à umidade (MARRA, 1992). A quantidade e a formulação da batida de cola variam em função das espécies utilizadas e propriedades requeridas. Matos (1997) produziu ao nível laboratorial, painéis LVL de Pinus taeda, com gramatura de $480 \mathrm{~g} / \mathrm{m}^{2}$ (linha dupla) e a seguinte formulação da batida de cola, em partes por peso: resina fenolformaldeído - 100, casca de noz em pó - 2, farinha de trigo 10 , água - 10 .

Objetivou-se, neste trabalho, avaliar a influência do uso de lâminas de Schizolobium amazonicum classificadas pelo método não destrutivo, e lâminas de Eucalyptus saligna e Pinus taeda nas capas, sobre as propriedades mecânicas de painéis laminados unidirecionais (LVL).

\section{MATERIAL E MÉTODOS}

\subsection{Material}

Foram utilizadas nesta pesquisa lâminas de madeiras de Schizolobium amazonicum (paricá) com 2,0 $\mathrm{mm}$ de espessura, coletadas numa indústria laminadora localizada no Município de Paragominas, estado do Pará.

Cerne, Lavras, v. 16, n. 4, p. 557-563, out./dez. 2010 
As lâminas de Eucalyptus saligna e Pinus taeda foram coletadas na indústria laminadora localizada no Município de Sengés, estado do Paraná. As massas específicas médias das lâminas das espécies em estudo foram respectivamente de $0,32 \mathrm{~g} / \mathrm{m}^{3}, 0,58 \mathrm{~g} / \mathrm{cm}^{3}$ e $0,42 \mathrm{~g} / \mathrm{cm}^{3}$, para Schizolobium amazonicum, Eucalyptus saligna e Pinus taeda. As lâminas sem defeitos, de acordo com os parâmetros de classificação visual, foram selecionadas e transportadas até o local de estudos.

Para a colagem de lâminas foi utilizada a resina fenolformaldeído, com teor de sólidos de 49\%, pH de 11,3 e viscosidade Brookfield de $420 \mathrm{cP}$. Demais componentes utilizados na batida de cola foram: farinha de trigo e farinha de casca de coco.

\subsection{Metodologia de pesquisa}

Os painéis laminados unidirecionais - LVL foram produzidos em escala laboratorial de acordo com o plano experimental apresentado na Tabela 1. Os painéis LVL foram produzidos com sete lâminas, todas elas dispostas na mesma direção da grã, utilizando-se as composições com lâminas de paricá selecionadas, aleatoriamente, e em três classes de MOEd, e lâminas de Pinus taeda e de Eucalytpus saligna nas faces (capa e contra-capa). Propôs-se, na pesquisa, avaliar a influência da resistência das lâminas, em diferentes composições sobre as propriedades mecânicas dos painéis.

Tabela 1 - Plano experimental.

Table 1 - Experimental chart.

\begin{tabular}{cl}
\hline Tratamento & \multicolumn{1}{c}{ Composição do painel } \\
\hline T1 & Lâminas selecionadas aleatoriamente \\
T2 & $\begin{array}{l}\text { Lâminas Pinus taeda capa / miolo paricá } \\
\text { aleatório }\end{array}$ \\
T3 & $\begin{array}{l}\text { Lâminas Eucalyptus saligna capa / miolo } \\
\text { paricá - aleatório }\end{array}$ \\
T4 & $\begin{array}{l}\text { Lâminas paricá - MOEd classe 1 } \\
\text { T5 }\end{array}$ \\
Lâminas paricá - MOEd classe 2 \\
T6 & $\begin{array}{l}\text { Lâminas paricá capa com MOEd classe 1/ } \\
\text { miolo classe 3 }\end{array}$ \\
\hline
\end{tabular}

As lâminas de madeira seccionadas com dimensões de $50 \times 50 \mathrm{~cm}$ foram secas em estufa, até o teor de umidade médio de $8 \%$. Conforme estabelecido no plano experimental, as lâminas de paricá foram pré-classificadas através do método não destrutivo com uso de strees-wave machine, em três classes de módulo de elasticidade dinâmico MOEd, para produção de painéis LVL com diferentes composições estruturais. As lâminas de Pinus taeda e Eucalyptus saligna foram utilizadas nas capas dos painéis nos tratamentos T2 e T3, respectivamente.

Para a colagem de lâminas foi utilizada a resina fenolformaldeído (FF) de acordo com a seguinte formulação em partes por peso: Resina FF (100), casca de coco (5), farinha de trigo (5) e água (5).

$\mathrm{O}$ adesivo foi aplicado manualmente com espátula sobre a superfície das lâminas com gramatura de $320 \mathrm{~g} / \mathrm{m}^{2}$ (superfície dupla). Os painéis foram prensados com pressão específica de $10 \mathrm{kgf} / \mathrm{cm}^{2}$, temperatura de $110^{\circ} \mathrm{C}$ e tempo de prensagem de 14 minutos. Foram produzidos dois painéis por tratamento, perfazendo um total de doze painéis para todo o experimento.

Após o processo de acondicionamento dos painéis na câmara climática, com temperatura de $20 \pm 2^{\circ} \mathrm{C}$ e umidade relativa de $65 \pm 5 \%$, foram retirados corpos de prova para realização dos seguintes ensaios em laboratório: (a) vinte corpos de prova para ensaios de resistência da linha de cola aos esforços de cisalhamento, sendo dez para teste seco e dez para fervura; (b) dez corpos de prova para ensaios de flexão estática para determinação de módulo de elasticidade (MOE) e módulo de ruptura (MOR), sendo cinco corpos de prova para direção paralela (edge) e cinco para direção perpendicular (flat) ao plano dos painéis. Os ensaios foram realizados de acordo com os procedimentos descritos na Norma Européia EN 314 e EN 310, respectivamente (EUROPEAN STANDARD, 2001, 2004).

Os resultados obtidos foram avaliados através da análise de variância e teste de Tukey ao nível de probabilidade de $95 \%$. O delineamento utilizado foi o inteiramente casualizado.

\section{RESULTADOS E DISCUSSÃO}

\subsection{Pré-classificação de lâminas de paricá com stress wave machine}

Na Tabela 2 estão apresentadas as faixas de módulo de elasticidade dinâmico MOEd e respectivas frequências das lâminas de paricá nas cinco classes definidas. Foram selecionadas as lâminas das classes 1, 2 e 3, com MOEd nas faixas de 3.001 a $4.000 \mathrm{MPa}, 4.001$ a $5.000 \mathrm{MPa}$ e 5.001 a $6.000 \mathrm{MPa}$, respectivamente, para composição dos painéis LVL conforme demonstrado no delineamento experimental.

Como base de referência, Matos (1997) obteve para lâminas de Pinus taeda com massa específica na faixa de 0,41 a $0,46 \mathrm{~g} / \mathrm{cm}^{3}$, valores de MOEd entre 7.354 a 8.578 
MPa. Neste estudo, as lâminas de paricá apresentaram menores valores de MOEd, porém a sua massa específica média de $0,32 \mathrm{~g} / \mathrm{cm}^{3}$ é bem inferior em comparação aos valores apresentados para o Pinus taeda.

Tabela 2 - Classes de MOEd das lâminas de paricá.

Table 2 - MOEd grades for paricá veneers.

\begin{tabular}{lcc}
\hline Classe & $\begin{array}{c}\text { Faixa de MOEd } \\
(\mathrm{MPa})\end{array}$ & $\begin{array}{c}\text { Frequência } \\
(\%)\end{array}$ \\
\hline Limite inferior & $<3.000$ & 2,0 \\
Classe 1 (MOEd 1) & 3.001 a 4.000 & 33,5 \\
Classe 2 (MOEd 2) & 4.001 a 5.000 & 32,88 \\
Classe 3 (MOEd 3) & 5.001 a 6.000 & 26,17 \\
Limite superior & $6.001>$ & 5,36 \\
\hline
\end{tabular}

MOEd: modulo de elasticidade dinâmico.

\subsection{Resistência da linha de cola aos esforços de cisalhamento}

Na Tabela 3, apresentam-se os valores médios de resistência da linha de cola aos esforços de cisalhamento (RLC) e porcentagens de falhas na madeira (FM), para os testes à seco e após fervura dos painéis LVL.

Os valores médios de resistência da linha de cola no teste seco (RLC) obtidos para os painéis LVL, com o miolo constituído de lâminas de paricá selecionadas aleatoriamente (T1 x T2 x T3), foram estatisticamente iguais entre si ao nível de probabilidade de $95 \%$. Entre os painéis produzidos com o miolo constituído de lâminas de paricá classificadas de acordo com o MOEd em classes 1, 2 e 3 (T4, T5 e T6), foram constatadas diferenças significativas apenas entre os tratamentos T4 e T6. Por esse resultado demonstra-se que a melhor qualidade de lâminas (MOEd 3) do tratamento T6, não contribuiu de forma positiva para o aumento na resistência da linha de cola.

Para os resultados obtidos nos testes de fervura não foram verificadas tendências claras dos efeitos da qualidade de lâminas de paricá sobre a resistência da linha de cola dos painéis LVL. As interações existentes entre a massa específica da madeira, a porosidade e a resistência mecânica das lâminas no processo de colagem de lâminas de paricá, não influenciaram de forma clara nos resultados de resistência da linha de cola, no teste de fervura.

Todos os resultados de RLC - após fervura, obtidos nesta pesquisa atendem ao requisito mínimo da Norma Européia EN 314-2 (1993), cujo valor é de 1,0 MPa, independente de percentagem de falhas na madeira.

\subsection{Flexão estática - Edge wise}

Na Tabela 4, apresentam-se os valores médios e respectivos coeficientes de variação para massa específica (ME), módulo de elasticidade (MOE) e módulo de ruptura (MOR), obtidos para o teste paralelo ao plano do painel (edge wise).

Os valores médios de massa específica dos painéis LVL produzidos integralmente com lâminas de paricá variaram na faixa de 0,372 a $0,414 \mathrm{~g} / \mathrm{cm}^{3}$. Já, para os painéis mistos, as inclusões de lâminas de Pinus taeda e de Eucalyptus saligna nas faces dos painéis LVL contribuíram para o aumento da sua massa específica. As maiores massas

Tabela 3 - Resultados de resistência da linha de cola aos esforços de cisalhamento, para os diferentes tratamentos.

Table 3 - Results of glue line shear strength for the different treatments.

\begin{tabular}{llllccc}
\hline \multirow{2}{*}{ Tratamento } & \multicolumn{3}{c}{ Teste seco } & \multicolumn{3}{c}{ Teste fervura } \\
\cline { 2 - 7 } & Média (MPa) & CV $(\%)$ & FM $(\%)$ & Média (MPa) & CV $(\%)$ & FM (\%) \\
\hline T1 - Pa/Ale & $1,81 \mathrm{~A}$ & 20,51 & 100 & $1,44 \mathrm{~A}$ & 16,09 & 94 \\
T2 - Pi-ca/Pa & $1,85 \mathrm{AB}$ & 16,67 & 100 & $1,81 \mathrm{BC}$ & 24,03 & 71 \\
T3 - Eu-ca/Pa & $2,05 \mathrm{ABC}$ & 15,80 & 100 & $1,99 \mathrm{C}$ & 18,12 & 96 \\
T4 - Pa/MOE1 & $2,47 \mathrm{C}$ & 25,28 & 100 & $2,02 \mathrm{C}$ & 14,61 & 81 \\
T5 - Pa/MOE2 & $2,31 \mathrm{BC}$ & 24,61 & 100 & $1,86 \mathrm{BC}$ & 11,19 & 75 \\
T6 - Pa-ca1/3 & $1,95 \mathrm{AB}$ & 21,25 & 100 & $1,67 \mathrm{AB}$ & 14,06 & 70 \\
\hline
\end{tabular}

Pa/Ale: Lâminas paricá selecionadas aleatoriamente; Pi-ca / Pa: Lâminas Pinus taeda capa / miolo paricá aleatório; Eu-ca / Pa: Lâminas Eucalyptus saligna capa / miolo paricá aleatório; Pa / MOE1: Lâminas paricá - MOEd classe 1; Pa / MOE2: Lâminas paricá MOEd classe 2; Pa-ca1/3: Lâminas paricá capa com MOEd classe 1 - miolo classe 3.

Médias com as mesmas letras na coluna não diferem estatisticamente, ao nível de probabilidade de $95 \%$.

Cerne, Lavras, v. 16, n. 4, p. 557-563, out./dez. 2010 
Tabela 4 - Resultados de massa específica (ME) e flexão estática - Edge wise para os diferentes tratamentos.

Table 4-Results of density and static bending tests - Edge wise for the different treatments.

\begin{tabular}{llllllc}
\hline \multirow{2}{*}{ Tratamento } & \multicolumn{2}{c}{ ME $\left(\mathrm{g} / \mathrm{cm}^{3}\right)$} & \multicolumn{2}{c}{ MOE-edge $(\mathrm{MPa})$} & \multicolumn{2}{c}{ MOR-edge $(\mathrm{MPa})$} \\
\cline { 2 - 6 } & Média & $\mathrm{CV}(\%)$ & Média & $\mathrm{CV}(\%)$ & Média & $\mathrm{CV}(\%)$ \\
\hline T1 - Pa/Ale & 0,404 & 4,23 & $20.102 \mathrm{C}$ & 12,12 & $74,5 \mathrm{~A}$ & 16,32 \\
T2 - Pi-ca/Pa & 0,500 & 4,50 & $15.624 \mathrm{~A}$ & 8,61 & $79,5 \mathrm{~A}$ & 11,86 \\
T3 - Eu-ca/Pa & 0,538 & 2,66 & $26.076 \mathrm{D}$ & 5,88 & $106,3 \mathrm{~B}$ & 6,97 \\
T4 - Pa/MOE1 & 0,414 & 2,64 & $19.605 \mathrm{BC}$ & 4,83 & $79,3 \mathrm{~A}$ & 10,50 \\
T5 - Pa/MOE2 & 0,372 & 4,09 & $17.241 \mathrm{AB}$ & 10,67 & $68,0 \mathrm{~A}$ & 13,12 \\
T6 - Pa-ca1/3 & 0,384 & 1,79 & $20.631 \mathrm{C}$ & 3,44 & $74,6 \mathrm{~A}$ & 6,95 \\
\hline
\end{tabular}

Ale: Lâminas selecionadas aleatoriamente; Pi-ca / Pa: Lâminas Pinus taeda capa / miolo paricá aleatório; Eu-ca / Pa: Lâminas Eucalyptus saligna capa / miolo paricá aleatório; Pa / MOE1: Lâminas paricá - MOEd classe 1; Pa / MOE2: Lâminas paricá - MOEd classe 2; Pa-ca1/3: Lâminas paricá capa com MOEd classe 1 - miolo classe 3.

Médias com as mesmas letras na coluna não diferem estatisticamente, ao nível de probabilidade de 95\%.

específicas das madeiras de Pinus taeda e Eucalyptus saligna resultaram em painéis com massa específica de $0,500 \mathrm{~g} / \mathrm{cm}^{3}$ e $0,538 \mathrm{~g} / \mathrm{cm}^{3}$, respectivamente.

A classificação de lâminas de paricá em três classes de MOEd não influenciaram, de forma clara, nos valores médios de módulo de elasticidade na direção paralela ao plano do painel (MOE-edge). O melhor resultado de MOEedge foi obtido para os painéis LVL produzidos com lâminas de Eucalyptus saligna nas faces superior e inferior (T3). A média obtida para esses painéis foi estatisticamente superior em relação aos demais tipos de painéis produzidos com lâminas de paricá e Pinus taeda nas faces (T2). Essa diferença pode ser atribuída à maior massa específica das lâminas de Eucalyputs saligna e sua disposição nas faces dos painéis, em que ocorrem maiores tensões de tração e de compressão nos ensaios de flexão estática.

Com relação ao módulo de ruptura na direção paralela ao plano do painel (MOR-edge), pelos resultados indicou-se igualdade estatística entre as médias obtidas para os painéis produzidos com diferentes composições de lâminas de paricá e de Pinus taeda nas faces (T2). Pelos painéis produzidos com lâminas de Eucalyptus saligna nas faces (T3) apresentou-se valor médio de MOR-edge, estatisticamente superior em comparação a todos os demais tipos de painéis. Mais uma vez, fica comprovada a influência da maior massa específica das lâminas de Eucalyptus saligna e sua disposição nas faces do painel, sobre os resultados de flexão estática.

Tanto para o MOE-edge, quanto para o MOR-edge, os resultados obtidos para os painéis LVL de paricá, indicaram que a pré-classificação de lâminas, com base no $\mathrm{MOE}$ dinâmico, não influencia de forma significativa nos resultados dessas propriedades. Essa avaliação é muito importante sobre o ponto de vista econômico, por ser um indicativo de não haver necessidade de realização dessa etapa do processo na produção de painéis LVL, utilizando-se lâminas de paricá. Provavelmente, a baixa massa específica das lâminas de paricá pode ter contribuído nos resultados obtidos, ou seja, a amplitude de variação é muito pequena a ponto de não justificar a classificação de lâminas, com base no módulo de elasticidade dinâmico.

\subsection{Flexão estática - Flat wise}

Na Tabela 5, apresentam-se os valores médios e respectivos coeficientes de variação para massa específica (ME), módulo de elasticidade (MOE) e módulo de ruptura (MOR), obtidos para o teste perpendicular ao plano do painel (flat wise).

Os valores médios de massa específica dos painéis LVL, que se produzem integralmente com lâminas de paricá variaram na faixa de 0,354 a $0,414 \mathrm{~g} / \mathrm{cm}^{3}$. Para os painéis mistos, com as inclusões de lâminas de Pinus taeda e de Eucalyptus saligna nas faces, houve aumento da massa específica para $0,450 \mathrm{~g} / \mathrm{cm}^{3}$ e $0,524 \mathrm{~g} / \mathrm{cm}^{3}$, respectivamente.

Os painéis LVL, produzidos com lâminas de Eucalyptus saligna nas faces superior e inferior (T3), apresentaram-se com valor médio de MOE-flat estatisticamente superior, em comparação a todos os tratamentos produzidos integralmente com lâminas de paricá e com lâminas de Pinus taeda nas faces. Por outro lado, a colagem de lâminas de Pinus taeda nas faces do

Cerne, Lavras, v. 16, n. 4, p. 557-563, out./dez. 2010 
Tabela 5 - Resultados de massa específica e flexão estática perpendicular - Flat wise.

Table 5 - Results of density and static bending tests (Flat wise).

\begin{tabular}{lcccccc}
\hline \multirow{2}{*}{ Tratamento } & \multicolumn{2}{c}{ ME $\left(\mathrm{g} / \mathrm{cm}^{3}\right)$} & \multicolumn{2}{c}{ MOE $(\mathrm{MPa})$} & \multicolumn{2}{c}{ MOR $(\mathrm{MPa})$} \\
\cline { 2 - 7 } & Média & $\mathrm{CV}(\%)$ & Média & CV $(\%)$ & Média & CV $(\%)$ \\
\hline T1 - Pa/Ale & 0,354 & 2,74 & $5.157 \mathrm{~B}$ & 5,19 & $26,4 \mathrm{~A}$ & 16,23 \\
T2 - Pi-ca/Pa & 0,450 & 5,83 & $3.944 \mathrm{~A}$ & 19,95 & $43,6 \mathrm{BC}$ & 23,91 \\
T3 - Eu-ca/Pa & 0,524 & 1,87 & $14.286 \mathrm{E}$ & 6,43 & $75,7 \mathrm{D}$ & 5,79 \\
T4 - Pa/MOE1 & 0,414 & 3,68 & $7.315 \mathrm{D}$ & 8,58 & $49,9 \mathrm{C}$ & 14,31 \\
T5 - Pa/MOE2 & 0,381 & 2,00 & $6.207 \mathrm{C}$ & 6,42 & $35,5 \mathrm{AB}$ & 8,59 \\
T6 - Pa-ca1/3 & 0,374 & 1,53 & $6.356 \mathrm{CD}$ & 6,05 & $36,9 \mathrm{~B}$ & 8,09 \\
\hline
\end{tabular}

Ale: Lâminas selecionadas aleatoriamente; Pi-ca / Pa: Lâminas Pinus taeda capa / miolo paricá aleatório; Eu-ca / Pa: Lâminas Eucalyptus saligna capa / miolo paricá aleatório; Pa / MOE1: Lâminas paricá - MOEd classe 1; Pa / MOE2: Lâminas paricá - MOEd classe 2; Pa-ca1/3: Lâminas paricá capa com MOEd classe 1 - miolo classe 3.

Médias com as mesmas letras na coluna não diferem, estatisticamente, ao nível de probabilidade de $95 \%$.

painel não contribuiu de forma significativa para o aumento do MOE-flat.

Os painéis constituídos com todas as lâminas de paricá da classse 1 (T4) apresentaram-se com média de MOE-flat, estatisticamente superior, em comparação aos painéis produzidos com lâminas de paricá da classe 2 (T5) e selecionadas aleatoriamente (T1). A composição do painel com lâminas de paricá da classe 1 nas faces e lâminas da classe 3 no miolo (T6) resultou em MOE-flat, estatisticamente igual, em comparação aos painéis produzidos integralmente com lâminas de paricá da classe 1 (T4) e classe 2 (T5). Por estes resultados aponta-se a disposição de lâminas de classe superior no miolo, não contribuiu para o aumento do MOE-flat, por se tratar de região da linha neutra onde são submetidas às baixas tensões de tração e de compressão.

Para o MOR- flat, da mesma forma como foi constatada para o MOE-flat, os painéis produzidos com lâminas de Eucalyptus saligna nas faces superior e inferior apresentaram-se com média estatisticamente superior em comparação a todos os tratamentos, produzidos integralmente com lâminas de paricá e com lâminas de Pinus taeda nas faces. A colagem de lâminas de Pinus taeda nas faces do painel não contribuiu de forma significativa para o aumento do MOR-flat em relação aos painéis produzidos com lâminas classificadas de paricá. O menor valor médio de MOR-flat foi obtido para os painéis produzidos com lâminas de paricá (T1), sem classificação com base no MOEd.

A maior massa específica das lâminas de Eucalyptus saligna e sua disposição nas faces dos painéis contribuíram de forma significativa para o aumento nos valores médios de MOE e MOR, tanto na direção paralela (edge), quanto na direção perpendicular ao plano do painel (flat).

Pelos ensaios de flexão estática indicam-se também que os valores médios obtidos para MOE e MOR, com a aplicação de carga na direção paralela ao plano do painel (Edge wise), são superiores em comparação aos valores médios de MOE e MOR na direção perpendicular ao plano do painel (Flat wise).

Em comparação com algumas fontes bibliográficas, os resultados que se obtiveram para o painel LVL de paricá, foram inferiores em relação aos valores médios de MOE e MOR-flat obtidos por Iwakiri et al. (2008) para painéis LVL de Eucalyptus grandis e Eucalyptus dunnii, cujos valores médios foram nas faixas de 12.764 a $18.310 \mathrm{MPa}$ e de 92,5 a $137,7 \mathrm{MPa}$, respectivamente. Entretanto, cabe ressaltar que essas espécies de eucalipto possuem massa específica bem superior em relação à madeira de paricá, o que contribuiu de forma significativa na maior resistência mecânica dos painéis LVL. Já, Matos (1997), obteve para painéis LVL de Pinus taeda, MOE e MOR-flat na faixa de 5.717 a 9.671 MPa e 29,5 a 44,4 MPa, respectivamente. Portanto, utilizando como base de referência as lâminas de Pinus taeda, pode-se afirmar que os painéis LVL produzidos com lâminas de Schizolobium amazonicum (paricá) demonstraram ser tecnicamente viáveis.

\section{CONCLUSÕES}

Com base nos resultados obtidos nesta pesquisa, as seguintes conclusões apresentam-se:

- A classificação de lâminas com base nas classes de MOEd não influenciou de forma significativa nos

Cerne, Lavras, v. 16, n. 4, p. 557-563, out./dez. 2010 
resultados de resistência da linha de cola de painéis LVL de paricá. Os resultados obtidos atendem aos requisitos mínimos da Norma Européia EN 314-2 (EUROPEAN STANDARD, 2004).

- A massa específica dos painéis LVL, produzidos com lâminas de paricá, foram bem inferiores em relação aos painéis compostos de lâminas de Eucalyptus saligna $e$ Pinus taeda nas faces.

- A classificação de lâminas de paricá com base nas classes de MOEd não influenciou de forma significativas nos resultados de MOE e MOR-edge.

- Para o MOE e MOR-flat, a classificação de lâminas de paricá com base nas classes de MOEd, contribuiu de forma significativa para o aumento nos valores médios dessas propriedades.

- Com a colagem de lâminas de Eucalyptus saligna nas faces do painel contribuiu-se para aumento nos valores médios de MOE e MOR, edge e flat.

- Os resultados de MOE e MOR, obtidos com a aplicação de carga na direção paralela ao plano do painel edge wise, foram bem superiores em comparação aos resultados obtidos para direção perpendicular ao plano do painel - flat wise.

- Os resultados de MOE e MOR demonstraram que, mesmo com a baixa massa específica da madeira de paricá, pode-se afirmar que a sua utilização na produção de painéis LVL é tecnicamente viável, tendo em vista que a sua performance foi similar aos painéis produzidos com lâminas de Pinus taeda nas faces.

\section{AGRADECIMENTOS}

Às empresas Selectas S.A. e Hexion Química Indústria e Comércio Ltda., pelas doações de lâminas de madeira e resina utilizadas nesta pesquisa.

\section{REFERÊNCIAS BIBLIOGRÁFICAS}

BALDWIN, R. F. Plywood and veneer-based products: manufacturing practices. San Francisco: M. Freeman, 1993. $388 \mathrm{p}$.

BENDSTEN, B. A. Properties of wood from improved and managed trees. In: ECONIMICS \& FINANCIAL MANAGEMENT AND TIMBER PRODUCTION TECHNICAL COMITTEES OF THE FOREST PRODUCTS RESEARCH SOCIETY, 1., 1978, Atlanta. Proceedings... Atlanta: FPRS, 1978. p. 78-121.
BODIG, J.; JAYNE, B. A. Mechanics of wood and wood composites. New York: V.N. Reinhold, 1982.711 p.

EUROPEAN STANDARD. EN 310: determination of modulus of elasticity in bending and of bending strength. Lisboa, 2002.

EUROPEAN STANDARD. EN 314-1: plywood, bonding quality, part 1: test methods. Bruxelas, 2004.

IWAKIRI, S. Painéis de madeira reconstituída. Curitiba: FUPEF, 2005. 247 p.

IWAKIRI, S.; MATOS, J. L. M.; TORQUATO, L. P.; BRONOSKI, M.; NISHIDATE, M. M. Produção de painéis laminados unidirecionais: LVL com madeira de Eucalyptus grandis Hill ex Maiden e Eucalyptus dunnii Maiden. Floresta e Ambiente, Seropédica, v. 15, n. 2, p. 1-7, 2008.

IWAKIR, S.; SHIMOYAMA, V. R. S.; PEDROSA, A. L.; ANJOS, R. A. M. Efeito do crescimento radial de Pinus taeda L. nas propriedades do painel compensado. Cerne, Lavras, v. 9, n. 1, p. 90-96, 2002.

MARRA, A. A. Technology of wood bonding: principles in practice. New York: V.N. Reinhold, 1992. 453 p.

MATOS, J. L. M. Estudos sobre a produção de painéis estruturais de lâminas paralelas de Pinus taeda L. 1997. 117 p. Tese (Doutorado em Ciências Florestais) - Universidade Federal do Paraná, Curitiba, 1997.

PEASE, D. A. Panels: products, applications and production trends. New York: M. Freeman, 1994. 254 p.

PIO, N. S. Produção de painéis estruturais de lâminas paraleleas (PLP) de Eucalyptus grandis Hill ex-Maiden. 2002. 174 p. Tese (Doutorado em Ciências Florestais) - Universidade Federal do Paraná, Curitiba, 2002.

SOCIEDADE BRASILEIRA DE SILVICULTURA. Fatos e números do Brasil Florestal 2006. São Paulo, 2007. Disponível em: 4http://www.sbs.org.brì Acesso em: 12 jan. 2009.

TSOUMIS, G. Science and technology of wood: stucture, properties and utilization. New York: V.N. Reinhold, 1991. $494 \mathrm{p}$.

Cerne, Lavras, v. 16, n. 4, p. 557-563, out./dez. 2010 
